\title{
THE VORTEX STRUCTURE OF LANGMUIR TURBULENCE IN THE INTERRUPTED MAGNETIC Z-PINCH. PART 1
}

\author{
P. Kubes ${ }^{1}$, A. K. Prykarpatsky ${ }^{2,3}$ \\ ${ }^{1}$ Dept. of Electrical Engineering, Czech Technical University, \\ 2 Technicna Str., 16627, Praha-6, Czech Republic \\ ${ }^{2}$ Dept. of Applied Mathem. at the AGH, Krakow 30-059, Poland \\ ${ }^{3}$ Dept. of Nonlinear Math. Analysis at the Institute for Applied Problems \\ of Mechanics and Mathematics of National Acad. Sci. of Ukraine, \\ 36 Naukova Str., Lviv, UA-290053, Ukraine \\ (Received May 20, 1997; received in final form May 6, 1998)
}

\begin{abstract}
The usual state of laboratory plasma is that of turbulence and as a consequence one needs to study its extreme characteristics among which the magnetic z-pinch structure appears to be very important for various technologies, sources of high temperature plasmas and local strong magnetic fields. In a turbulence the energy of the system is shared between very many different modes. If some characteristic time-scales are provided, it then becomes possible to describe the turbulence by kinetic equations governing the transfer of energy between the modes. In this study we shall restrict ourselves to the so called Langmuir turbulence, that is the turbulence dominated by the behaviour of the longitudinal electromagnetic or Langmuir modes, leading to the creation of the vortex structure in the interrupted magnetic $\mathrm{z}$-pinch. This vortex structure is stable enough for a spheroidal plasmoid to exist for a rather long time as it was discovered in recent experiments. Since physical processes giving rise to such a structure are essentially nonlinear, one needs to derive from the very basic principles the corresponding vortex generating equations and to treat them under the conditions at the experiments. As a result we have built the so called paired Hill's vortex solutions compatible with the ambient magnetic field supplying the wanted stability condition of the plasmoid existence at the magnetic $\mathrm{z}$-pinch.
\end{abstract}

Key words: vortex, plasmoid structure, z-pinch.

PACS numbers: $52.55 . \mathrm{Ez}$

\section{THE VORTEX EQUATIONS AND PLASMOID STRUCTURE}

We shall first derive the necessary equations from a two-fluid description of the plasma as this provided a clear picture of interactions, following Zakharov [4] and ter Haar [1].

Let us write the ion density $n_{i}$ and the electron density $n_{e}$ in the form

$$
n_{i}=n_{0}+\delta n_{i}, \quad n_{e}=n_{0}+\delta n_{s}+\delta n_{f},
$$

where $\delta n_{i}$ and $\delta n_{s}$ vary on the slow time-scale $\tau_{i} \geq$ $\omega_{p i}^{-1}=\left(m_{i} / 4 \pi n_{0} e^{2}\right)^{1 / 2}$, and $\delta n_{f}$ on the fast time-scale $\tau_{e} \leq \omega_{p e}^{-1}=\left(m_{e} / 4 \pi n_{0} e^{2}\right)^{1 / 2} ; n_{0}$ is the equilibrium density, $m_{e}$ - the electron mass, $m_{i}$ - the ion mass and $e$ - the electron charge.

On the slow time-scale the average of $\delta n_{f}$ vanishes:

$$
\left\langle\delta n_{f}\right\rangle=0
$$

where $\langle\cdot\rangle$ denotes the temporal averaging operation. Similarly, one can write the electron fluid velocity $\mathbf{v}_{e}$ in the form:

$$
\mathbf{v}_{e}=\mathbf{v}_{s}+\mathbf{v}_{f}
$$

The high mobility of electrons as compared to that of the ions ensures approximate charge quasi-neutrality on the ion time-scale, or

$$
\delta n_{s} \approx \delta n_{i} \equiv \delta n, \quad \mathbf{v}_{s} \sim \mathbf{v}_{i} \equiv \mathbf{v}
$$

but the symbols have been kept distinct, as there are small differences between them which drive ion-sound oscillations.

We shall consider the following equations in what follows as basic:

(i) Maxwell equations for free charges:

$$
\begin{aligned}
& \operatorname{div} \mathbf{E}=4 \pi \rho, \quad \operatorname{rot} \mathbf{E}=-\frac{1}{c} \frac{\partial \mathbf{B}}{\partial t}, \\
& \operatorname{div} \mathbf{B}=0, \quad \operatorname{rot} \mathbf{B}=\frac{4 \pi}{c} \mathbf{j}+\frac{1}{c} \frac{\partial \mathbf{E}}{\partial t},
\end{aligned}
$$

whence we get

$$
\frac{\partial^{2} \mathbf{E}}{\partial t^{2}}+c^{2} \operatorname{rot} \operatorname{rot} \mathbf{E}=-4 \pi \frac{\partial \mathbf{j}}{\partial t}
$$


The charge density $\rho$ and the current density $\mathbf{j}$ are given by equations

$$
\rho=-e\left(n_{e}-n_{i}\right), \quad \mathbf{j}=-e\left(n_{e} \mathbf{v}_{e}-n_{i} \mathbf{v}_{i}\right)
$$

(ii) the viscousless electron-fluid equation of motion:

$$
\begin{aligned}
\frac{\partial \mathbf{v}_{e}}{\partial t} & +\left(\mathbf{v}_{e} \cdot \nabla\right) \mathbf{v}_{e}+\frac{e}{m_{e}}\left(\mathbf{E}+\frac{\mathbf{v}_{e}}{c} \times \mathbf{B}\right) \\
& +\frac{\gamma_{e} T_{e}}{n_{e} m_{e}} \nabla n_{e}=0
\end{aligned}
$$

(iii) the viscousless ion-fluid equation of motion:

$$
\begin{aligned}
\frac{\partial \mathbf{v}_{i}}{\partial t} & +\left(\mathbf{v}_{i} \cdot \nabla\right) \mathbf{v}_{i}-\frac{e}{m_{i}}\left(\mathbf{E}+\frac{\mathbf{v}_{i}}{c} \times \mathbf{B}\right) \\
& +\frac{\gamma_{i} T_{i}}{n_{i} m_{i}} \nabla n_{i}=0,
\end{aligned}
$$

where the values of the specific heat ratios $\gamma_{e} \simeq 3$ and $\gamma_{i} \simeq 1$ are not essential in our further investigation. The action of the electrons on the ions and vice versa is mediated by the slow part of the electronic field and does not appear explicitly.

We need further to split the electronic and magnetic fields, the charge density, the current density and the electron velocity into their slow and fast parts:

$$
\begin{gathered}
\mathbf{E}=\mathbf{E}_{s}+\mathbf{E}_{f}, \quad \mathbf{B}=\mathbf{B}_{s}+\mathbf{B}_{f}, \\
\mathbf{j}=\mathbf{j}_{s}+\mathbf{j}_{f}, \quad \rho=\rho_{s}+\rho_{f} .
\end{gathered}
$$

From (7) one can find that

$$
\mathbf{j}_{f}=-e\left(n_{0}+\delta n_{s}\right) \mathbf{v}_{f}-e\left[\delta n_{f} \mathbf{v}_{f}-\left\langle\delta n_{f} \mathbf{v}_{f}\right\rangle\right]
$$

where the harmonic generating term in square brackets can be dropped in what will follow. Taking the fast part component of eq. (9), we obtain that

$$
\begin{aligned}
& \frac{\partial \mathbf{v}_{f}}{\partial t}+\left(\mathbf{v}_{s} \cdot \nabla\right) \mathbf{v}_{f}+\left(\mathbf{v}_{f} \cdot \nabla\right) \mathbf{v}_{s}+\left[\left(\mathbf{v}_{f} \cdot \nabla\right) \mathbf{v}_{f}-\left\langle\mathbf{v}_{f} \cdot \nabla \mathbf{v}_{f}\right\rangle\right. \\
& \left.\left.+\frac{e}{m_{e}} \mathbf{E}_{f}+\frac{\mathbf{v}_{f}}{c} \times \mathbf{B}_{s}+\frac{\mathbf{v}_{s}}{c} \times \mathbf{B}_{f}-\left\langle\frac{\mathbf{v}_{f}}{c} \times \mathbf{B}_{f}\right\rangle\right)+\frac{\mathbf{v}_{f}}{c} \times \mathbf{B}_{f}\right]-\frac{\gamma_{e} T_{e}}{4 \pi n_{0} m_{e} e} \nabla\left(\operatorname{div} \mathbf{E}_{f}\right)=0
\end{aligned}
$$

where we have used Poisson's equation in the last term and in the denominator of that term neglected $\delta n_{s}$ as compared to $n_{0}$. As a result of omitting all convective terms one can get that

$$
\begin{aligned}
\frac{\partial^{2} \mathbf{E}_{f}}{\partial t^{2}} & +\omega_{p e}^{2}\left(\mathbf{E}_{f}+\frac{\mathbf{v}_{f}}{c} \times \mathbf{B}_{s}+\frac{\mathbf{v}_{s}}{c} \times \mathbf{B}_{f}\right)+c^{2} \operatorname{rot} \operatorname{rot} \mathbf{E}_{f}+\frac{3 T_{e}}{m_{e}} \nabla\left(\operatorname{div} \mathbf{E}_{f}\right) \\
& =\frac{\delta n_{s}}{n_{0}} \omega_{p e}^{2}\left(\mathbf{E}_{f}+\frac{\mathbf{v}_{f}}{c} \times \mathbf{B}_{s}+\frac{\mathbf{v}_{s}}{c} \times \mathbf{B}_{f}\right)
\end{aligned}
$$

and

$$
\frac{\partial \mathbf{v}_{s}}{\partial t}+\left(\mathbf{v}_{s} \cdot \nabla\right) \mathbf{v}_{s}+\left(\mathbf{v}_{f} \cdot \nabla\right) \mathbf{v}_{f}+\frac{e}{m_{e}} \times\left(\mathbf{E}_{s}+\frac{\mathbf{v}_{s}}{c} \mathbf{B}_{s}+\left\langle\mathbf{v}_{f} \times \mathbf{B}_{f}\right\rangle\right)+\frac{\gamma_{e} T_{e}}{n_{0} m_{e}} \nabla\left(\delta n_{e}\right)=0
$$

Taking into account now the lowest order on the last time scale we can obtain from (12) that

$$
\frac{\partial \mathbf{v}_{f}}{\partial t} \simeq-\frac{e}{m_{e}}\left(\mathbf{E}_{f}+\frac{\mathbf{v}_{f}}{c} \times \mathbf{B}_{s}+\frac{\mathbf{v}_{s}}{c} \times \mathbf{B}_{f}\right)
$$

which combined with the Maxwell equations gives

$$
\frac{\partial}{\partial t}\left(\operatorname{rot} \mathbf{v}_{f}-\frac{e}{c m_{e}} \mathbf{B}_{f}\right) \simeq 0
$$

$\mathbf{B}_{f} \simeq\left(c m_{e} / e\right)$ rot $\mathbf{v}_{f}$ in accordance with the condition $\operatorname{div} \mathbf{B}_{f} \equiv 0$. As a result we can find that 


$$
\begin{gathered}
\frac{\partial \mathbf{v}_{s}}{\partial t}+\left(\mathbf{v}_{s} \cdot \nabla\right) \mathbf{v}_{s}+\frac{e}{m_{e}}\left(\mathbf{E}_{s}+\frac{\mathbf{v}_{s}}{c} \times \mathbf{B}_{s}\right) \\
+\nabla\left(\frac{\gamma_{e} T_{e}}{n_{0} m_{e}} \delta n_{s}+\frac{\left\langle\mathbf{E}_{f}^{2}\right\rangle}{m_{e} 8 \pi n_{0}}\right)=0 \\
\frac{\partial \mathbf{v}_{i}}{\partial t}+\left(\mathbf{v}_{i} \cdot \nabla\right) \mathbf{v}_{i}-\frac{e}{m_{i}}\left(\mathbf{E}_{s}+\frac{\mathbf{v}_{s}}{c} \times \mathbf{B}_{s}\right) \\
+\nabla\left(\frac{\gamma_{i} T_{i}}{n_{0} m_{i}} \delta n_{i}+\frac{m_{e}}{m_{i}^{2} 8 \pi n_{0}}\left\langle\mathbf{E}_{f}^{2}\right\rangle\right)=0 .
\end{gathered}
$$

Equations (13), (17) and (18) together with the Maxwell equations (5) lead us to the main object of studying the Langmiur turbulence of a plasma. To come for a vortex structure equations, let us take the rot-operation to the left hand sides of eqs. (17) and (18):

$$
\begin{aligned}
& \frac{\partial \boldsymbol{\Omega}_{s}}{\partial t}+\operatorname{rot}\left(\boldsymbol{\Omega}_{s} \times \mathbf{v}_{s}\right)=0, \\
& \frac{\partial \boldsymbol{\Omega}_{i}}{\partial t}+\operatorname{rot}\left(\boldsymbol{\Omega}_{i} \times \mathbf{v}_{i}\right)=0,
\end{aligned}
$$

where by definition, we set

$$
\begin{aligned}
& \boldsymbol{\Omega}_{s}:=\operatorname{rot}\left(\mathbf{v}_{s}-\frac{e}{c m_{e}} \mathbf{A}_{s}\right), \\
& \boldsymbol{\Omega}_{i}:=\operatorname{rot}\left(\mathbf{v}_{i}+\frac{e}{c m_{i}} \mathbf{A}_{s}\right), \\
& \operatorname{rot} \mathbf{A}_{s}:=\mathbf{B}_{s} .
\end{aligned}
$$

Therefore, we can assert that into each plasma component there are frozen the corresponding vortex vectors $\Omega_{s}$ and $\Omega_{i}$.

\section{THE PLASMOID STRUCTURE}

We proceed now to considering a plasma moving between two electrodes generating via a discharge a structure of the interrupted $\mathrm{z}$-pinch.

Let us now consider the structure of the vortex vectors $\Omega_{s}$ and $\Omega_{i}$, taking into account the Maxwell equations $(5)$ :

$$
\operatorname{rot} \mathbf{j}_{s} \simeq-e n_{0}\left(\operatorname{rot} \mathbf{v}_{s}-\operatorname{rot} \mathbf{v}_{i}=-e n_{0}\left(\boldsymbol{\Omega}_{s}+\frac{\mathbf{B}_{s} e}{m_{e} c}-\Omega_{i}+\frac{\mathbf{B}_{s} e}{c m_{i}}\right)=e n_{0}\left(\boldsymbol{\Omega}_{s}-\boldsymbol{\Omega}_{i}\right)-\frac{e^{2} n_{0}}{c}\left(\frac{1}{m_{e}}+\frac{1}{m_{i}}\right) \mathbf{B}_{s}\right.
$$

whence,

$$
\begin{aligned}
\frac{4 \pi e n_{0}}{c}\left(\boldsymbol{\Omega}_{s}-\boldsymbol{\Omega}_{i}\right) & =-\frac{4 \pi}{c} \operatorname{rot} \mathbf{j}_{s}-\frac{4 \pi e^{2} n_{0}\left(m_{i}+m_{e}\right)}{c^{2} m_{e} m_{i}} \mathbf{B}_{s}-\operatorname{rot} \operatorname{rot} \mathbf{B}_{s}-\frac{4 \pi n_{0}\left(m_{i}+m_{e}\right)}{c^{2} m_{e} m_{i}} \mathbf{B}_{s} \\
& +\frac{1}{c} \operatorname{rot} \frac{\partial \mathbf{E}_{s}}{\partial t} \simeq \triangle \mathbf{B}_{s}-\frac{4 \pi e^{2} n_{0}}{c^{2} m_{e}} \mathbf{B}_{s}-\frac{1}{c^{2}} \frac{\partial^{2} \mathbf{B}_{s}}{\partial t^{2}}
\end{aligned}
$$

Thus we obtain from (19) and (21) that

$$
\frac{1}{c^{2}} \frac{\partial^{2} \mathbf{B}_{s}}{\partial t^{2}}+\frac{\omega_{p e}^{2}}{c^{2}} \mathbf{B}_{s}-\triangle \mathbf{B}_{s}=\boldsymbol{\xi}
$$

where $\xi=\mu_{0} e n_{0}\left(\Omega_{i}-\Omega_{s}\right)$.

The expressions (21) and (22) lead us to the following final equations on the vectors $\boldsymbol{\xi}, \Omega_{i}$ and $\boldsymbol{\Omega}_{s}$ :

$$
\begin{aligned}
& -\triangle \mathbf{B}_{s}+\frac{\omega_{p e}^{2}}{c^{2}} \mathbf{B}_{s}+\frac{1}{c^{2}} \frac{\partial^{2} \mathbf{B}_{s}}{\partial t^{2}}=\boldsymbol{\xi}, \\
& \frac{\partial}{\partial t} \boldsymbol{\Omega}_{s}+\operatorname{rot}\left(\boldsymbol{\Omega}_{s} \times \mathbf{v}_{s}\right)=0, \\
& \frac{\partial}{\partial t} \boldsymbol{\Omega}_{i}+\operatorname{rot}\left(\boldsymbol{\Omega}_{i} \times \mathbf{v}_{i}\right)=0,
\end{aligned}
$$

where $\omega_{p e}^{2}:=\left(4 \pi e^{2} n_{0} / m_{e}\right)^{1 / 2}$ — the so called plasmon frequency. In case of incompressibility of the plasma under consideration one can transform equations (23) into the following ones having used the axis-symmetry of the magnetic 
$\mathrm{z}-$ pinch [3]:

$$
\begin{aligned}
& \frac{D}{D t}\left(\frac{\Omega_{i, \theta}}{r}\right)=0, \\
& \frac{D}{D t}\left(\frac{\Omega_{s, \theta}}{r}\right)=0,
\end{aligned}
$$

where $\Omega_{i}=\left(0, \Omega_{i, \theta}, 0\right), \Omega_{s}=\left(0, \Omega_{s, \theta}, 0\right) \in \mathbf{R}^{3}, D / D t$ - the corresponding material (Lagrangian) derivative. We will use the cylindrical coordinate system $(r, \theta, z) \in$ $\mathbf{R}_{+} \times[0,2 \pi) \times \mathbf{R}$ and we also put $\boldsymbol{\xi}:=\left(0, \xi_{\theta}, 0\right), \mathbf{B}_{s}:=$ $\left(0, \mathbf{B}_{\theta}, 0\right) \in \mathbf{R}^{3}$, where we have taken into account the actual absence of either turning round motion in the plasma. Incompressibility of the plasma makes it possible to present the velocity components $v_{i, z}, v_{i, r}$ and $v_{s, z}, v_{s, \theta}$ as follows:

$$
\begin{aligned}
& v_{s, r}:=\frac{1}{r} \frac{\partial \Psi_{s}}{\partial z}, \quad v_{s, z}:=-\frac{1}{r} \frac{\partial \Psi_{s}}{\partial r} \\
& v_{i, r}:=\frac{1}{r} \frac{\partial \Psi_{i}}{\partial z}, \quad v_{i, z}:=-\frac{1}{r} \frac{\partial \Psi_{i}}{\partial r}
\end{aligned}
$$

where $\Psi_{i}(r, z), \Psi_{s}(r, z)$ - some potential functions.

As a result we obtain that the functions $\Psi_{i}(r, z)$,
$\Psi_{s}(r, z)$ satisfy the following equations:

$$
\begin{aligned}
& \frac{D}{D t}\left(\frac{\boldsymbol{\Omega}_{s, \theta}}{r}\right)=0, \quad \frac{D}{D t}\left(\frac{\boldsymbol{\Omega}_{i, \theta}}{r}\right)=0 \\
& \boldsymbol{\Omega}_{i, \theta}=\frac{1}{r}\left(\frac{\partial^{2} \Psi_{i}}{\partial r^{2}}-\frac{1}{r} \frac{\partial \Psi_{i}}{\partial r}+\frac{\partial^{2} \Psi_{i}}{\partial z^{2}}\right)-\frac{e}{m_{i}} \mathbf{B}_{s, \theta} \\
& \boldsymbol{\Omega}_{s, \theta}=\frac{1}{r}\left(\frac{\partial^{2} \Psi_{s}}{\partial r^{2}}-\frac{1}{r} \frac{\partial \Psi_{s}}{\partial r}+\frac{\partial^{2} \Psi_{s}}{\partial z^{2}}\right)-\frac{e}{m_{e}} \mathbf{B}_{s, \theta}
\end{aligned}
$$

The simplest Hill's solution [5] to eq. (26) is given by the following completely stationary expressions:

$$
\begin{aligned}
& \mathbf{B}_{s, \theta}=C_{\theta} r, \quad \Omega_{i, \theta}=\frac{15 Z_{i} r}{2 a^{2}}-C_{\theta} r \frac{e}{m_{i}}, \quad 0 \leq r \leq a \\
& \boldsymbol{\Omega}_{s, \theta}=\frac{15 Z_{s} r}{2 a^{2}}-C_{\theta} r \frac{e}{m_{e}} \\
& \mathbf{B}_{s, \theta}=0, \quad \Omega_{i, \theta} \equiv 0, \quad \Omega_{s, \theta}=0, \quad r \geq a
\end{aligned}
$$

where $Z_{i}$ and $Z_{s} \in \mathbf{R}$ and $a \in \mathbf{R}_{+}$- some arbitrary constants. Then from (26) one can exact the solution $\Psi(r, z)$ as follows:

$$
\begin{aligned}
& \Psi_{i}(r, z)=r^{2}\left\{\frac{3 Z_{i}}{4 a^{2}}\left(r^{2}-a^{2}\right)+\frac{3 Z_{i}}{4 a^{2}}\left(z-Z_{i}\right)^{2}-\frac{1}{2} Z_{i}\right\} \\
& \Psi_{s}(r, z)=r^{2}\left\{\frac{3 Z_{s}}{4 a^{2}}\left(r^{2}-a^{2}\right)+\frac{3 Z_{s}}{4 a^{2}}\left(z-Z_{s}\right)^{2}-\frac{1}{2} Z_{s}\right\} .
\end{aligned}
$$

The solution (28) and its derivatives with respect to $z$ and $r$ are compatible at $r \rightarrow a \pm 0$ with the corresponding solution to (26) at $z \in \mathbf{R}, r \geq a$, when $\xi_{\theta} \equiv 0$. From (25) and (28) one can get the following dynamic characteristics of the plasma flow:

$$
\begin{aligned}
& v_{i, r}=3 Z_{i} r\left(z-Z_{i}\right) / 2 a, v_{s, r}=3 Z_{s} r\left(z-Z_{s}\right) / 2 a, \quad 0 \leq r \leq a, \\
& v_{i, r}=3 a^{2} Z_{i} r\left(z-Z_{i}\right) / 2 R_{i}^{5}, v_{i, r}=3 a^{2} Z_{s} r\left(z-Z_{s}\right) / 2 R_{s}^{5}, \quad r \geq a ; \\
& v_{i, z}=Z_{i}\left[5 a^{2}-3\left(z-Z_{i}\right)^{2}-6 r^{2}\right] / 2 a^{2}, \quad 0 \leq r \leq a, \\
& v_{s, z}=Z_{s}\left[5 a^{2}-3\left(z-Z_{s}\right)^{2}-6 r^{2}\right] / 2 a^{2}, \\
& v_{i, z}=a^{3} Z_{i}\left[3\left(z-Z_{i}\right)^{3}-R_{i}^{2}\right] / 2 R_{i}^{5}, \quad r \geq a, \\
& v_{s, z}=a^{3} Z_{s}\left[3\left(z-Z_{s}\right)^{3}-R_{s}^{2}\right] / 2 R_{s}^{5},
\end{aligned}
$$

where $R_{i}^{2}:=r^{2}+\left(z-Z_{i}\right)^{2}, R_{s}^{2}:=r^{2}+\left(z-Z_{s}\right)^{2}$.

The solution (29) describes a spherical vortex in the plasma moving along the axis $O z$ with the constant velocity $Z=$ const, this vortex being completely contained inside a sphere of a radius $a>0$.

It is obvious that the spherical vortex part of solution (29) can model the plasmoid creation existing during the interrupted z-pinch in a plasma, described in article [3]. To justify the above conclusion, we need to agree solution (27) for the velocity $\xi_{\theta}$ with the corresponding magnetic field equation of (22). Thus we must solve the following magnetic field equations for the magnetic field $\mathbf{B}_{s}:=\left(B_{r}, B_{\theta}, B_{z}\right) \in \mathbf{R}^{3}, \triangle Z=Z_{i}-Z_{s}, k=\left(m_{i}+m_{e}\right) / m_{i} m_{e}$ : 


$$
\begin{aligned}
& \triangle B_{\theta}-\frac{\omega_{p e}^{2}}{c^{2}} B_{\theta}-\frac{1}{c^{2}} \frac{\partial^{2} B_{\theta}}{\partial t^{2}}=e n_{0} \mu_{0} \frac{15 Z r}{2 a^{2}}-k C_{\theta} r \mu_{0} e n_{0}, 0 \leq r \leq a, \\
& \triangle B_{\theta}-\frac{\omega_{p e}^{2}}{c^{2}} B_{\theta}-\frac{1}{c^{2}} \frac{\partial^{2} B_{\theta}}{\partial t^{2}}=0 \quad r \geq a
\end{aligned}
$$

with the solenoidal condition $\operatorname{div} \mathbf{B}_{s} \equiv 0$. The last two equations plausibly would lead us to a possibility of conforming the vortex structure of a plasmoid from paper [3] where this hypothesis was claimed too. Thereby we can formulate the following theorem.

Theorem The dynamic vortex structure (26), (28) describes a plasmoid generated by the interrupted $z$-pinch [3] in a plasma if and only if the solenoidal magnetic field system (30) is compatible for all $r \geq 0$ at some fixed $p a-$ rameters $a>0, \triangle Z \neq 0$ and

$$
\begin{aligned}
C_{\theta} & =15 \triangle Z e n_{0} /\left[2 a^{2}\left(k e n_{0}-\omega_{p e}^{2} \varepsilon_{0}\right)\right] \\
& \cong-15 \triangle Z e n_{0} /\left(2 a^{2} \omega_{p i}^{2} \varepsilon_{0}\right)
\end{aligned}
$$

Note. Below we rewrite our equations (19) and (23) in the following somewhat generalized continual form:

$$
\begin{aligned}
B_{t t}+\omega_{p e}^{2} B & =c^{2} \triangle B+\mu_{0} c^{2} \operatorname{rot} j, \\
E & =-v \times B+2 J \\
\omega_{t} & +\operatorname{rot}(\omega \times v) \\
& =-e n_{0} k B_{t}-e n_{0} k \operatorname{rot}(B \times v)+\nu \triangle \omega, \\
v_{t} & +\operatorname{rot} v \times v \\
& +\frac{1}{n_{0} m_{i}} \nabla\left(p+\frac{v^{2}}{2}\right)-J \times B+v \triangle \nu=0,
\end{aligned}
$$

where $\omega=\operatorname{rot} j, v=v_{s}, B=B_{s}, \nu \in \mathbf{R}_{+}$-the viscosity and $p \in \mathbf{C}^{\infty}\left(\mathbf{R}^{3} ; \mathbf{R}\right)$-some external and intrinsic ponderomotive pressure in the plasma flow. Equations (31) are obtained basing on the averaging procedure on the ion time-scale. They can be extended still with the pressure equation $[7,9]$ :

$$
\begin{aligned}
& \frac{1}{\gamma-1}\left(\frac{\partial p}{\partial t}+\left(v_{i} \nabla\right) p\right)=\gamma j^{2}+2 \nu \sum_{i, j=1}^{3} e_{i j} e_{j i} \\
& e_{i j}=\frac{1}{2}\left(\frac{\partial v_{i}}{\partial x_{j}}+\frac{\partial v_{j}}{\partial x_{i}}\right)
\end{aligned}
$$

where $\gamma=5 / 3$ - the specific ion heat, $\eta \simeq 1 \cdot 10^{-4}$ the resistivity. The resulting systems (31) and (32) can be studied also by numerical calculations giving rise to driven magnetic reconnections of the Hill type spheromarks [7] playing an important role in explanation of the interrupted magnetic $\mathrm{z}$-pinch in a plasma.

The next important question arises whether the solution obtained above is stable under some small perturbations of the corresponding boundary and initial data. Fortunately this can be studied in detail and solved affirmatively due to the Hamiltonian structure of vorticity equations (23) as shown in [6]. On some problems important for modelling the plasmoid structure we are going to stay in detail in Part 2 of the article.

\section{ACKNOWLEDGEMENT}

The authors are grateful to Dr. J. Zagrodzinski, Dr. J. Stakhira and Dr. D. Blackmore for helpful discussions. This research was supported by grants GACR No. 202-95-0178 "Stable Structures in Magnetic Pinches" and No. 202-97-0487 "X-ray Source on the Magnetic Pinch Principle".
[1] S. G. Thornhill, ter D. Haar, Phys. Rep. C 43, 43 (1978).

[2] L. I. Rudakov, V. N. Tsytovich, Phys. Rep. C 401 (1978).

[3] P. Kubes, J. Kravarik, J. Hakr, P. Kulhanek, J. Pichal, IEEE Trans. Plasma Sci. 22, 986 (1994).

[4] V. E. Zakharov, Sov. Phys. JETP 35, 908 (1972).

[5] M. J. M. Hill, Philos. Trans. R. Soc. London A 185, 213 (1894).

[6] D. D. Holm, J. E. Marsden, T. Ratiu, A. Weinstein, Phys. Rep. 123, 1 (1985).
[7] J. Gibbon, Physica D 3, 503 (1981).

[8] N. N. Bogoliubov, V. H. Samoilenko, A. K. Prykarpatsky, Ukr. Fiz. Zh. 37, 147 (1992).

[9] S. S. Moiseev, R. E. Sagdeev, A. V. Tur, V. V. Yanovsky, Probliemy sil'noi turbulientnosti i topologichieskiie solitony: nielinieinyie yavlieniia $v$ plazmie $i$ gidrodinamikie (The problems of strong turbulence and topological solitons: Nonlinear Phenomena in Plasma and Hydrodynamics) (Moscow, Mir, 1986), p. 137. 


\title{
ВИХРОВА СТРУКТУРА ТУРБУЛ ЕНЩЇ Л ЕНГМЮРА В ПЕРЕРВАНОМУ $\mathrm{Z}-$ ПIНЧІ. ЧАСТИНА 1
}

\author{
П. Кубеш ${ }^{1}$, А. К. Прикарпатський ${ }^{2,3}$ \\ ${ }^{1}$ Факультет електричной інженерій, Чесъкий технічний університет, \\ вул. Технічна, 2, Прага-6, 16627, Чесъка республіка \\ E-mail: kubes@feld.cvut.cz \\ ${ }^{2}$ Факультет прикладнӧ̈математики АГХ, Краків 30-059, Польща \\ ${ }^{3}$ відділ нелінійного математичного аналізу ІППММ НАН Украйни, \\ вул. Наукова, 3б, Львів, 290053, Украӥна \\ E-mail:prykan@cybergal.com
}

Одним із станів, у якому може перебувати плазма в лабораторних умовах, є стан із турбулентністю; тому виникає проблема вивчення екстремальних характеристик турбулентногостану. Серед них структура магнетного z-пінчу відіграє ва жливу роль для різних технологій та джерел високотемпературної плазми і локальних сильних магнетних полів. При турбуленції енергія системи розділяється мі ж багатьма різними модами. При умові забезпечености певної часової характеристики турбуленція описується кінетичними рівняннями. У нашому розгляді ми обмежимось так званою турбуленцією Ленгмюра, яка приводить до створення вихрової структури в перерваному z-пінчі. Стабільна для сферичного плазмоїда вихрова структура існує перева жно довгий час, як це було показано в недавніх експериментах. Оскільки фізичні процеси, що приводять до такої структури, є нелінійними, потрібно отримати з загальних принципів відповідні рівняння для вихорів та розв'язати іх згідно з умовами експерименту. Як результат ми маємо побудувати пару розв'язків Гілла, сумісну з магнетним полем, що забезпечує шукані умови стабільности для існування плазми при магнетному z-пінчі. 